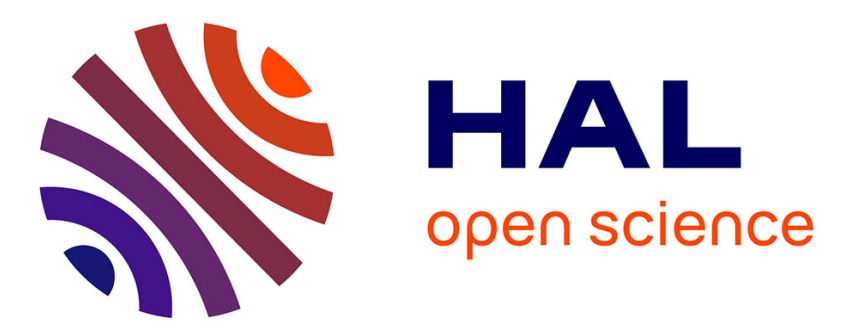

\title{
Periodic saltation over hydrodynamically rough beds: aeolian to aquatic
}

Diego Berzi, James Thomas Jenkins, Alexandre Valance

\section{To cite this version:}

Diego Berzi, James Thomas Jenkins, Alexandre Valance. Periodic saltation over hydrodynamically rough beds: aeolian to aquatic. Journal of Fluid Mechanics, 2016, 786, pp.190- 209. 10.1017/jfm.2015.601 . hal-01231830

\section{HAL Id: hal-01231830 https://hal.science/hal-01231830}

Submitted on 28 Jan 2016

HAL is a multi-disciplinary open access archive for the deposit and dissemination of scientific research documents, whether they are published or not. The documents may come from teaching and research institutions in France or abroad, or from public or private research centers.
L'archive ouverte pluridisciplinaire HAL, est destinée au dépôt et à la diffusion de documents scientifiques de niveau recherche, publiés ou non, émanant des établissements d'enseignement et de recherche français ou étrangers, des laboratoires publics ou privés. 


\title{
Periodic saltation over hydrodynamically rough beds: Aeolian to aquatic
}

\author{
Diego Berzi ${ }^{1}$, James T. Jenkins ${ }^{2}$ and Alexandre Valance ${ }^{3}$ \\ ${ }^{1}$ Department of Civil and Environmental Engineering, Politecnico di Milano, 20133 Milano, Italy \\ ${ }^{2}$ School of Civil and Environmental Engineering, Cornell University, Ithaca, NY 14850, USA \\ ${ }^{3}$ Institut de Physique de Rennes, Université de Rennes I, 35042 Rennes, France
}

\begin{abstract}
We determine approximate, analytical solutions for average, periodic trajectories of particles that are accelerated by the turbulent shearing of a fluid between collisions with a hydrodynamically rough bed. We indicate how the viscosity of the fluid may influence the collisions with the bed. The approximate solutions compare well with periodic solutions for average periodic trajectories over rigid-bumpy and erodible beds that are generated numerically. The analytic solutions permit the determination of the relations between the particle flux and the strength of the shearing flow over a range of particle and fluid properties that vary between those for sand in air and sand in water.
\end{abstract}

\section{Introduction}

The transport of sediments by the shearing of a turbulent fluid over a rigid or an erodible bed is an important phenomenon in many civil and industrial applications. Despite several decades of investigation on this topic, its description largely relies on empirical formulations, and a complete theory in which the various physical mechanisms are treated in a transparent way is lacking. Indeed, sediment transport consists of different regimes, each characterized by a different mode of transport and therefore, by a different physics.

If, for simplicity, we focus on spheres of uniform size and mass, the strength of the shearing flow controls the mode of transport. Below a certain threshold, the forces that the fluid transmits to the particles are not sufficient to set them in motion. Above the threshold, a few erratic particles are seen to slide, roll and jump, in an intermittent way, associated with the local burst of turbulence (Ancey et al. 2008, Drake et al. 1988, Lajeunesse et al. 2010, Nelson et al. 1995, Radice et al. 2009).

If the strength of the shearing flow increases, the number of moving particles increases as well, and they move by successive jumps over the bed without intermediate periods of rest. This regime, called saltation, is the primary mode of transport in Aeolian sand transport, and has been investigated theoretically (Bagnold 1941, Bagnold 1966, Owen 1964, Ungar \& Haff 1987, Sauermann et al. 2001, Andreotti 2004, Jenkins et al. 2010), experimentally (Bagnold 1941, Nalpanis et al. 1993, Foucaut \& Stanislas 1997, Iversen \& Rasmussen 1999, Ho et al. 2014) and numerically (Anderson \& Haff 1988, Kok \& Renno 2009). More references can be found in recent reviews on Aeolian transport (Durán et al. 2011, Kok et al. 2012, Valance et al. 2015). Saltation may also be relevant in aquatic flows (Fernandez Luque \& van Beek 1976, Abbot \& Francis 1977, Ancey et al. 2002, Niño \& García 1998). The differences in saltation occur because of the difference in the mass density of the material of the grains relative to that of air and water and the differences in the kinematic viscosities of the two fluids. Saltation of grains of other mass densities in other atmospheres and other gravities is possible and has been described (Burr et al. 2015, Greeley et al. 1984, Iversen \& White 1982, White 1979).

Stronger shearing flows make inter-particle collisions above the bed probable, and these collisions provide a mechanism to sustain the weight of the particles (Berzi \& Fraccarollo 2013, Jenkins \& Hanes 1998, Pasini \& Jenkins 2005). At even stronger shearing, the weight of the particles is counter-balanced by the mean turbulent lift - turbulent suspension (Drew 1975, McTigue 1981, Hsu et al. 2003). If the particle sizes and/or masses are poly-dispersed, the different regimes may coexist at a given strength of the shearing flow. 
Here, we focus on continuing saltation, defined as the regime in which the mean motion of the fluid is strong enough to cause continuing motion of the particles through successive jumps between collisions with the bed; the probability of having collisions above the bed is negligible, and the interaction of the particles with the bed is crucial in determining the characteristics of the jumps. Particularly relevant to continuing saltation, in the range of parameters between sand particles in air and water, are the discrete numerical simulations of Durán et al. (2012). They consider identical spheres in two dimensions that interact with a turbulent shearing flow above a dense aggregate of spheres with which they collide. In their simulations, they measured average flow quantities and could infer from their measurements how these scaled with the strength of the shearing flow and the properties of the particles and fluid. Jenkins and Valance (2014) obtained simpler solutions of average, periodic, Aeolian saltation trajectories and were able to reproduce and extend the relations determined by Durán et al. (2012). Jenkins and Valance (2014) considered sand particles in air that are accelerated by the wind between collisions with either a rigid, bumpy bed or an erodible, particle bed. Collisions were characterized by coefficients of restitution for the total and vertical components of the velocity that depend on the geometry of the collision.

A collision of a particle with a rigid, bumpy bed involves only its rebound. At a given strength of the wind, the particle flux associated with the trajectories over a rigid bed exhibits a maximum when plotted against the mass of particles per unit area above the bed - the mass hold-up. That is, for a given particle flux, there are two possible mass hold-ups; of these two, that for which the flux decreases with mass holdup is conjectured to be unstable. Collisions with erodible beds often involve both the rebound of the colliding particle and the ejection of others. Experiments that involve a single impacting particle show that there is a critical impact velocity below which there is only rebound and no ejection (Beladjine et al. 2007). In a natural flow over a particle bed, there is a distribution of impact velocities below the critical velocity. In our analysis of steady flows, we assume that this distribution and the mass hold-up adjust, so that the maximum possible value of the particle flux for the given wind speed is attained.

Here, the solutions of periodic saltation trajectories are further extended to more general systems than sand in air on Earth and, more importantly, approximate analytical solutions of the trajectory equations are derived. They permit the determination of the dependence of the flow quantities on the strength of the turbulent shearing, gravity, and the properties of the grains and the fluid. The analytical solution agrees well with the numerical solutions of the periodic trajectories over rigid beds. For flows over erodible beds, we extend the criterion of Jenkins and Valance (2014), based on the average of a uniform distribution, for the determination of the unique particle flux associated with a given strength of the shearing flow. This permits the prediction of the evolution of scaling of the particle flux with the strength of the shearing flow from Aeolian to aquatic saltation, as first seen by Durán et al. (2012) in their numerical simulations.

The paper is organized as follows. The equations governing the periodic motion of the particles and the fluid flow are described in Section 2. The analytical solutions for the rigid and the erodible bed case are introduced in Section 3 and 4, respectively. Conclusions are drawn in Section 5. 


\section{Trajectory equations}

We briefly summarize the equations, already introduced by Jenkins and Valance (2014), that govern the periodic saltating motion of a sphere of diameter $d$ and mass density $\rho^{s}$ over a horizontal bed. A turbulent shearing flow of a fluid of mass density $\rho^{f}$ and molecular viscosity $\mu^{f}$ drives the flow in the presence of gravity, with $g$ the gravitational acceleration. The horizontal velocity of the turbulent fluid is $U$, and the horizontal and vertical components of the particle velocity are $\xi_{x}$ and $\xi_{y}$, respectively. We assume that the flow is steady and uniform, so that the velocities are only functions of the vertical distance from the bed $y$. The flow configuration is depicted in Fig. 1.

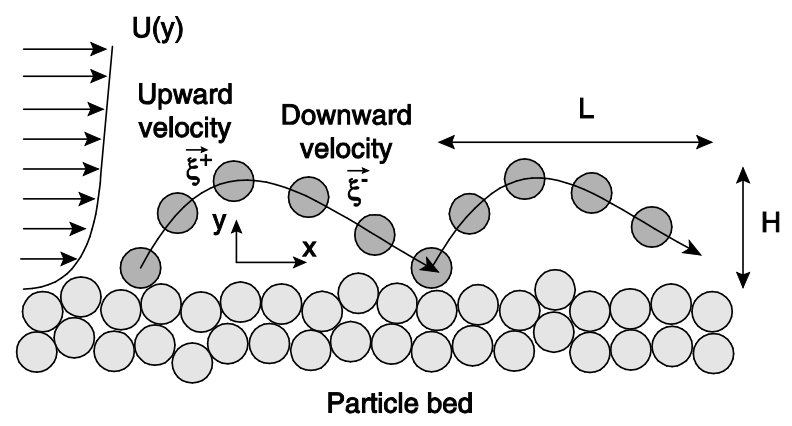

Figure 1. Sketch of the periodic trajectory.

We characterize the particles through the fall particle Reynolds number $\mathrm{R}=\rho^{f} \sqrt{g(\sigma-1) / \sigma} d^{3 / 2} / \mu^{f}$ , where $\sigma=\rho^{s} / \rho^{f}$ is the density ratio. All quantities are made dimensionless

using the diameter and mass density of the particle and the reduced gravitational acceleration $g(\sigma-1) / \sigma$. We characterize the drag exerted on the particles through a nonlinear drag coefficient, which is, in dimensionless form,

$$
D=\frac{0.3}{\sigma} \sqrt{\left(U-\xi_{x}\right)^{2}+\xi_{y}^{2}}+\frac{18}{\mathrm{St}},
$$

where $\mathrm{St}=\sigma \mathrm{R}$

is a Stokes number. In the following, we use the subscripts plus and minus to label

quantities in the upward and downward parts of a trajectory, respectively.

The upward and downward components of the particle velocity are governed by

$$
\begin{gathered}
\xi_{y}+\frac{\mathrm{d} \xi_{x}^{+}}{\mathrm{d} y}=D^{+}\left(U-\xi_{x}^{+}\right), \\
\xi_{y} \frac{\mathrm{d} \xi_{y}^{+}}{\mathrm{d} y}=-1-D^{+} \xi_{y}^{+},
\end{gathered}
$$

and

$$
\xi_{y}^{-} \frac{\mathrm{d} \xi_{x}^{-}}{\mathrm{d} y}=D^{-}\left(U-\xi_{x}^{-}\right),
$$




$$
\xi_{y} \frac{\mathrm{d} \xi_{y}^{-}}{\mathrm{d} y}=-1-D^{-} \xi_{y}^{-},
$$

respectively. The horizontal coordinates $x^{+}$and $x$ are functions of the vertical coordinate $y$,

$$
\xi_{y}^{+} \frac{\mathrm{d} x^{+}}{\mathrm{d} y}=\xi_{x}^{+},
$$

and

$$
\xi_{y}^{-} \frac{\mathrm{d} x^{-}}{\mathrm{d} y}=\xi_{x}^{-}
$$

The average particle velocity $u$, and the particle shear stress $s$ are

$$
u \equiv \frac{c^{+} \xi_{x}^{+}+c^{-} \xi_{x}^{-}}{c^{+}+c^{-}}
$$

and

$$
s \equiv-\left(c^{+} \xi_{y}^{+} \xi_{x}^{+}+c^{-} \xi_{y}^{-} \xi_{x}^{-}\right),
$$

where $c$ is the particle concentration. In steady, uniform saltation, the vertical mass fluxes of ascending and descending particles must balance at any point, and must be independent of the vertical position. Hence,

$$
c^{+} \xi_{y}^{+}=-c^{-} \xi_{y}^{-}=\phi_{0}
$$

where $\phi_{0}$ is the vertical mass flux at the bed. The upward flux is sometimes referred to as the pick-up function.

The fluid shear stress, $S$, is the difference between the dimensionless fluid shear stress far from the bed, $S^{*}$ - the Shields parameter - and the particle shear stress: $S=S^{*}-s$. In this paper, we restrict our attention to hydrodynamically rough beds; so, upon employing Prandtl's mixing length hypothesis, the horizontal velocity of the turbulent fluid is given in terms of $S$ by

$$
\frac{\mathrm{d} U}{\mathrm{~d} y}=\frac{(\sigma S)^{1 / 2}}{\kappa\left(y+y_{0}\right)}
$$

where $\kappa=0.41$ is Karman's constant and $y_{0}$ is the dimensionless roughness length, here taken to be $1 / 30$ (Jenkins \& Valance 2014, Van Rijn 1984). The assumption that the bed is rough requires that $\mathrm{R}\left(\sigma S^{*}\right)^{1 / 2}$ larger than 70 (Gersten \& Schlichting 2000); then the thickness of the viscous sub-layer is much smaller than the particle diameter. 
The system of Eqs. 2 through 7 and 11 permits the determination of the seven unknowns $\xi_{x}^{+}, \xi_{y}^{+}, \xi_{x}$, $y^{-}, x^{+}, x$ and $U$, after the application of seven boundary conditions. The latter are the kinematic relations $x^{+}(0)=0^{\prime} x^{-}(0)=L^{\prime} x^{+}(H)=x^{-}(H)^{\prime} \xi_{x}^{+}(H)=\xi_{x}^{-}(H)^{\prime} \xi_{y}^{+}(H)=\xi_{y}^{-}(H)=0{ }^{\text {, where } H \text { and } L \text { are the }}$ height and the length of the periodic particle trajectory, and the no-slip condition

$$
U(0)=0 . \text { When two }
$$

further boundary conditions are introduced, the trajectory height and length can be determined as part of the solution. Experiments and numerical simulations (Beladjine et al. 2007, Crassous et al. 2007, Oger et al. 2005) have shown that the particles rebound at a bumpy bed according to

$$
\xi_{0}^{+}=e \xi_{0}^{-}
$$

where the subscript 0 indicates evaluation at the bed, and

$$
\xi_{y 0}^{+}=-e_{y} \xi_{y 0}^{-}
$$

where $\xi \equiv \sqrt{\xi_{x}^{2}+\xi_{y}^{2}}$ is the magnitude of the particle velocity. In the absence of significant damping due to the presence of a viscous fluid, $e \equiv a-b \sin \theta \stackrel{\text { and }}{e_{y} \equiv a_{y} / \sin \theta-b_{y}}$, in which $a, b, a_{y}$ and $b_{y}$ are numerical constants that depend on the coefficients of normal restitution and sliding friction of the particles and whether the bed is rigid or erodible (Beladjine et al. 2007, Crassous et al. 2007, Oger et al. 2005) and $\theta$ is the angle between the bed and the incident trajectory:

$$
\tan \theta \equiv-\xi_{y 0}^{-} / \xi_{x 0}^{-}
$$

. In what follows, we use $a=0.87$,

$b=0.72, a_{y}=0.30$ and $b_{y}=0.15$ for both the impact of spheres on a rigid, bumpy bed and on a threedimensional bed of like spheres (Beladjine et al. 2007). Also, we indicate the modifications of these expressions to account for viscous dissipation in the way suggested by Yang and Hunt (2006), and already implemented to predict collisional sediment transport (Berzi \& Fraccarollo 2013). In this case, the coefficients of restitution are assumed to depend on the Stokes number, so that

$$
\xi_{0}^{+}=e \xi_{0}^{-}-\frac{62(1+e)}{\mathrm{St}}
$$

and

$$
\xi_{y 0}^{+}=-e_{y} \xi_{y 0}^{-}-\frac{62\left(1+e_{y}\right)}{\mathrm{St}}
$$

For saltation over rigid, bumpy beds, there are no additional conditions, so that the solution is determined by the specification of two control parameters: the Shields parameter, $S^{*}$, and the upward flux $\phi_{0}$. The latter can be equivalently replaced by the mass hold-up, as in Jenkins \& Valance (2014); or by the horizontal particle flux $Q=\phi_{0} L$, as in the experiments (Ho et al. 2011); or by the vertical velocity after the 
rebound $\xi_{y 0}^{+}$, the take-off velocity, as in the present work. In any case, there is a range of possible particle

fluxes that can be steadily sustained at a given strength of the fluid flow.

For saltation over erodible beds, experiments (Creyssels et al. 2009, Meyer-Peter \& Müller 1948) and numerical simulations (Durán et al. 2012) show that there is only one particle flux associated with a given Shields parameter, no matter what the density ratio. In the formulation of the periodic boundaryvalue problem, that means that there is an additional constraint that permits the determination of the upward flux - or equivalently the horizontal particle flux - as part of the solution. Trajectories over erodible beds often involve both the rebound of the colliding particle and the ejection of others. Collision experiments with a single particle show that there is a critical impact velocity, $\xi_{c}$, below which there is only rebound and no ejection (Beladjine et al. 2007, Crassous et al. 2007, Oger et al. 2005). Hence, in order to have steady saltation over erodible beds, no incoming particle velocity can exceed $\xi_{c}$, with the latter roughly equal to 40 (Beladjine et al. 2007). In case of a uniform distribution of impacting velocities less than $\xi_{c}$, the average value of the impacting velocities must be less than $\xi_{c} / 2$. Jenkins \& Valance (2014) were, indeed, able to reproduce the experiments on Aeolian saltation over erodible beds using the additional constraint that the impacting velocity of their periodic trajectory was always equal to $\xi_{c} / 2$. We will show how the assumption of a range of impact velocities less than $\xi_{c} / 2$ permits the prediction of the qualitative different relationships between the horizontal particle flux and the Shields parameter in Aeolian and aquatic transport over erodible beds.

Finally, we note that, the critical impact velocity depends on the amount of energy transferred and dissipated in successive collisions between the particles in the bed that are initially at rest and in contact with each other (Ho et al. 2012). When an interstitial fluid is present, we assume that it does not play a role in determining the value of $\xi_{c}$.

\section{Approximate analytical solution: periodic saltation over a rigid, bumpy bed}

Periodic trajectories over a rigid, bumpy bed exist for a range of impact velocity. So, as already mentioned, in order to determine the numerical solution to the periodic trajectory over a rigid, bumpy bed, we must prescribe both the Shields parameter and another parameter that quantifies the flow of particles in the system. Here, we take this to be the take-off velocity. This choice, although formally correct, might seem unusual, because the take-off velocity cannot be controlled in a physical experiment. However, the parameterization of the problem in terms of the take-off velocity permits the significant insight that the periodic particle trajectories and the fluid shear stress at the bed are, essentially, independent of the Shields parameter, which enters only in the determination of the particle shear stress and concentration.

\subsection{Single particle trajectories}

We first determine the steady periodic trajectories that are compatible with the rebound relations Eqs. 14 and 15. If we assume that the vertical velocity after the rebound is so small that the drag in the vertical momentum balances can be neglected, the trajectory height is simply

$$
H=\frac{1}{2}\left(\xi_{y 0}^{+}\right)^{2}
$$

and the downward vertical velocity before impacting the bed is

$$
\xi_{y 0}^{-}=-\xi_{y 0}^{+}
$$


Figure 2 shows the results of the numerical integration for the periodic trajectories at different values of the Stokes number and density ratio obtained for different values of the Shields parameter. Equation 16 gives the upper envelope of the numerical results - the height is maximum when the vertical drag is negligible. We note that the vertical drag causes a significant reduction in the heights of the trajectories from that of Eq. 16 for small density ratios, Stokes numbers less than 1000 and take-off velocities larger than five.

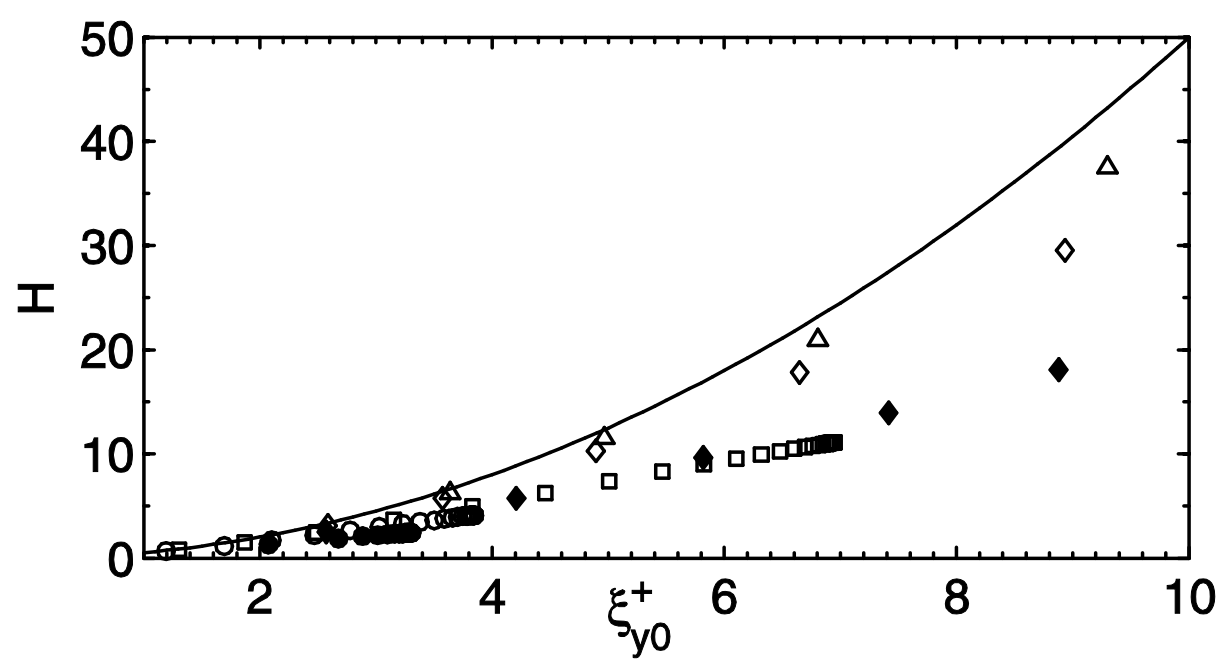

Figure 2. Trajectory height versus take-off velocity obtained from numerical solutions of the periodic trajectories: St $=1000$ (hollow symbols) and St $=100$ (solid symbols), when $\sigma=5$ (circles), $\sigma=10$ (squares), $\sigma=100$ (diamonds) and $\sigma=1000$ (triangles). The solid line is the approximate analytical solution of Eq. 16.

For Stokes numbers as large as several hundred, we can ignore the dependence of the coefficients of restitution on the Stokes number, so that

$$
\begin{aligned}
& e_{y}=1^{\prime} \tan \theta \simeq \sin \theta \simeq a_{y} /\left(1+b_{y}\right) \text { and } \\
& \xi_{x 0}^{-}=\frac{1+b_{y}}{a_{y}} \xi_{y 0}^{+}=A \xi_{y 0}^{+} .
\end{aligned}
$$

For arbitrary values of the Stokes number, $e_{y}$ is determined from Eq. 15 as indicated in Appendix A. For large Stokes numbers, the impact velocity is, from Eqs. 17 and 18,

$$
\xi_{0}^{-}=\left[\left(\frac{1+b_{y}}{a_{y}}\right)^{2}+1\right]^{1 / 2} \xi_{y 0}^{+} \equiv B \xi_{y 0}^{+} .
$$

Figure 3 shows the comparison between the numerical solutions for the periodic trajectories for different values of the Shields parameter and the impact velocity predicted by Eq. 19. The latter reproduces the numerical results for Stokes number larger than several hundred, with no influence of the density ratio. When the Stokes number is 100 , there is a departure of the numerical results from the analytical prediction; this is associated with the influence of the viscosity on the rebound. When this effect is included in the approximate analytical solution (Appendix $A$ ) the agreement is also good when $\mathrm{St}=100$. For small Stokes numbers, the dependence of the numerical results on the density ratio is due to the vertical drag (Fig. 2). Finally, when the effect of the viscosity is included in the rebound relations, the impact velocity has a minimum, and this minimum increases with the Stokes number. 


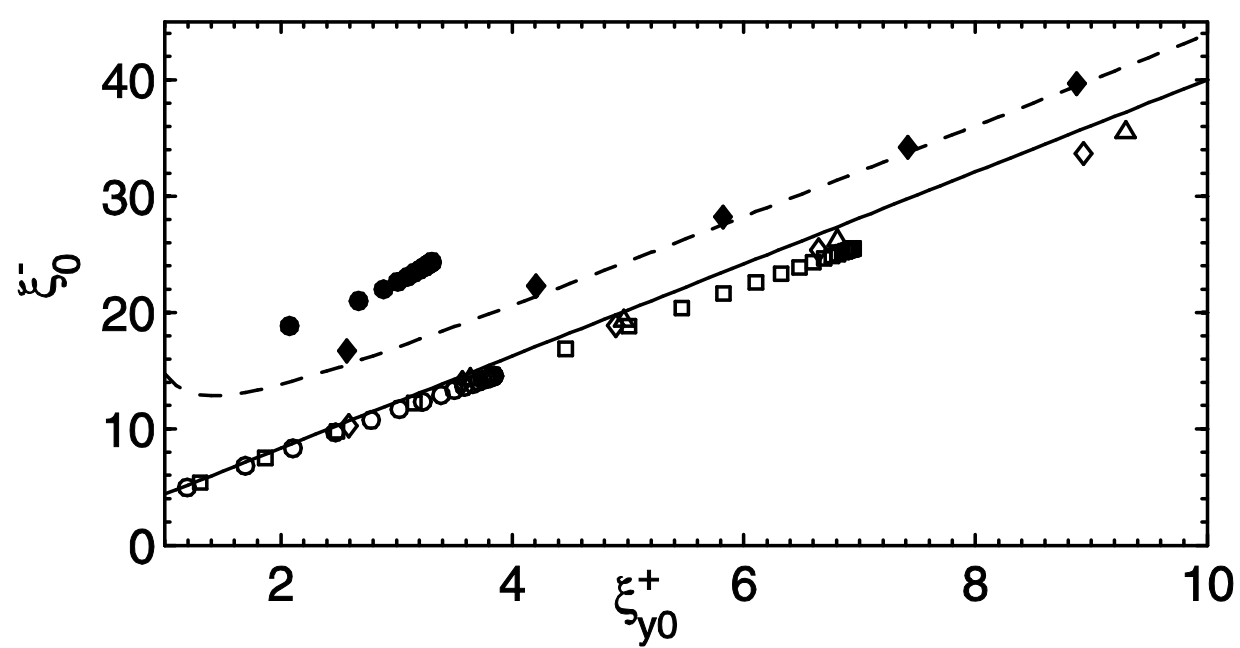

Figure 3. Absolute value of the impact velocity versus take-off velocity obtained from the numerical solutions for the periodic trajectories. The symbols are the same as in Fig. 2 . The solid line is the analytical approximation in the limit of large Stokes number of Eq. 19; the dashed line is the analytical approximation with the dependence on the Stokes number retained (Eq. A5).

From Eqs. 14 and 19, the absolute value of the velocity after the rebound is, for large Stokes $\xi_{0}^{+}$

numbers,

$$
\xi_{0}^{+}=\left(a-b \frac{a_{y}}{1+b_{y}}\right)\left[\left(\frac{1+b_{y}}{a_{y}}\right)^{2}+1\right]^{1 / 2} \xi_{y 0}^{+} \equiv C \xi_{y 0}^{+}
$$

Hence, the horizontal particle velocity after the rebound is

$$
\xi_{x 0}^{+}=\left\{\left(a-b \frac{a_{y}}{1+b_{y}}\right)^{2}\left[\left(\frac{1+b_{y}}{a_{y}}\right)^{2}+1\right]-1\right\}^{1 / 2} \xi_{y 0}^{+} \equiv D \xi_{y 0}^{+} .
$$

\subsection{Fluid velocity}

We next integrate the horizontal particle momentum balances, assuming that the fluid velocity is equal to its depth-averaged over the trajectory height ${ }_{\bar{U}}$. Equation 4 may then be re-written as

$$
\frac{\mathrm{d} \xi_{x}}{\mathrm{~d} t}=\frac{0.3}{\sigma}\left(\bar{U}-\xi_{x}\right)^{2}+\frac{18}{\mathrm{St}}\left(\bar{U}-\xi_{x}\right)
$$

where $t$ is time, and we have taken

$$
\bar{U}-\xi_{x} \gg \xi_{y} \text {. Upon Integrating, }
$$




$$
\frac{\bar{U}-\xi_{x 0}^{+}}{\bar{U}-\xi_{x}} \frac{0.3\left(\bar{U}-\xi_{x}\right) / \sigma+18 / \mathrm{St}}{0.3\left(\bar{U}-\xi_{x 0}^{+}\right) / \sigma+18 / \mathrm{St}}=\exp \left(\frac{18}{\mathrm{St}} t\right)
$$

The uniform fluid velocity is determined from Eq. 23, using Eqs. 18 and 21, and the fact that the time of flight in a ballistic trajectory is equal to $2 \xi_{y 0}^{+}$. It is worth noting that large Stokes numbers do not affect the collisions with the bed, but do influence the fluid velocity. For Stokes numbers as large as several hundred, the approximate result is

$$
\bar{U}=\frac{A+D}{2} \xi_{y 0}^{+}+E
$$

where

$$
E \equiv[(A-D) / 0.6]^{1 / 2} \sigma^{1 / 2}-18 \sigma /(0.6 \mathrm{St})
$$

and we have neglected a term proportional to $\left[(A-D) \xi_{y 0}^{+}\right]^{2}$

In this approximation, the depth-averaged fluid velocity is linearly related to the particle

take-off velocity. The quantity $E$ can be interpreted as the excess in the depth-averaged horizontal fluid velocity over the mean of the horizontal particle velocities before and after the impact, which is needed to sustain the particle motion. Figure 4 shows that the uniform fluid velocity obtained from Eq. 24 is proportional to the horizontal fluid velocity, depth-averaged through the trajectory height, measured in the numerical solutions for the periodic trajectories. The latter have been obtained for different values of the Shields parameter, given the Stokes number and the density ratio; hence, Fig. 4 also shows that the depthaveraged fluid velocity is independent of the strength of the fluid flow, a rather counterintuitive result.

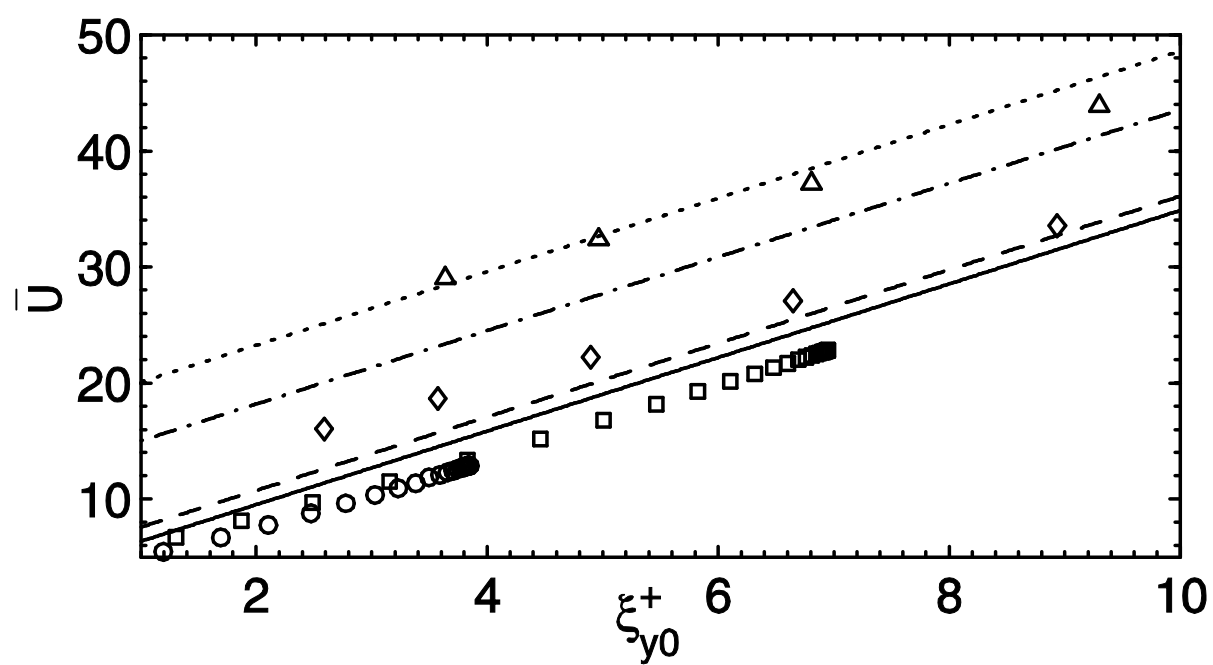

Figure 4. Horizontal fluid velocity, depth-averaged over the trajectory height, obtained from the numerical solutions for the periodic trajectories at large Stokes numbers versus take-off velocity. The symbols are the same as in Fig. 2 . The lines are the analytical approximations (Eq. 24) when: $\mathrm{St}=1000$ and $\sigma=5$ (solid), $\sigma=10$ (dashed), $\sigma=100$ (dotdashed) and $\sigma=1000$ (dotted).

\subsection{Trajectory length}

Equation 23 then provides the particle horizontal velocity at every instant $t$. Upon integrating, 


$$
\begin{aligned}
x= & \bar{U} t+\frac{\sigma}{0.3} \frac{18}{\mathrm{St}} t-\frac{\sigma}{0.3} \ln \left[\exp \left(\frac{18}{\mathrm{St}} t\right)-\frac{0.3 \mathrm{St}\left(\bar{U}-\xi_{x 0}^{+}\right)}{0.3 \mathrm{St}\left(\bar{U}-\xi_{x 0}^{+}\right)+18 \sigma}\right] \\
& +\frac{\sigma}{0.3} \ln \left[1-\frac{0.3 \mathrm{St}\left(\bar{U}-\xi_{x 0}^{+}\right)}{0.3 \mathrm{St}\left(\bar{U}-\xi_{x 0}^{+}\right)+18 \sigma}\right] .
\end{aligned}
$$

If we employ Eq. 24 in this and take $t=2 \xi_{y 0}^{+}$, we can calculate the trajectory length. The result is well approximated by

$$
L \simeq(A+D) \xi_{y 0}^{+2}
$$

as demonstrated in Fig. 5.

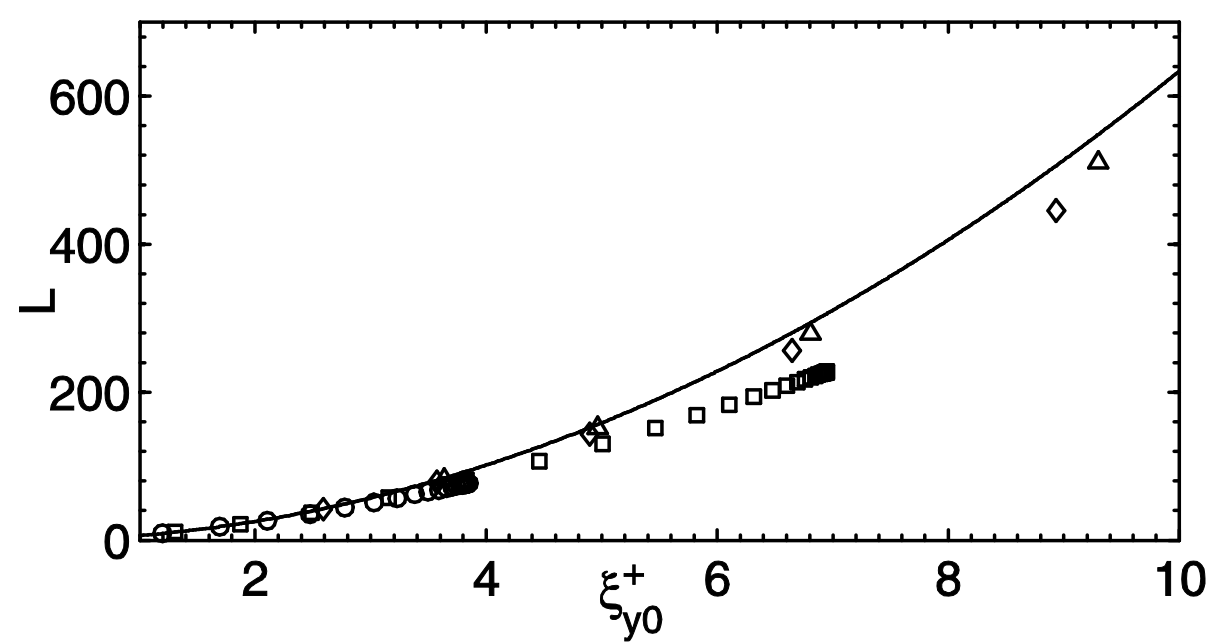

Figure 5. Trajectory length versus take-off velocity at large Stokes numbers. The symbols are the same as in Fig. 3 . The solid line is the analytical approximation (Eq. 26) to the trajectory length.

\subsection{Fluid shear stress}

If the horizontal fluid velocity follows the turbulent logarithmic profile based on the roughness, we can calculate the fluid shear stress at the bed, $S_{0}$, as (Appendix B)

$$
S_{0}=K \frac{\kappa^{2}}{\sigma\left[\ln \left(H / y_{0}\right)\right]^{2}} \bar{U}^{2},
$$

where $K$ is a coefficient of order one, which we determine on the basis of comparisons with the full numerical solution of the equations for the periodic trajectories. With $K=1.5$, the agreement with the results of the numerical solution is good (Fig. 6). Once again, the fluid shear stress depends only on the take-off velocity and is independent of $S^{*}$, so the numerical solution to the periodic trajectories for different Shields parameters collapse onto a single curve. 


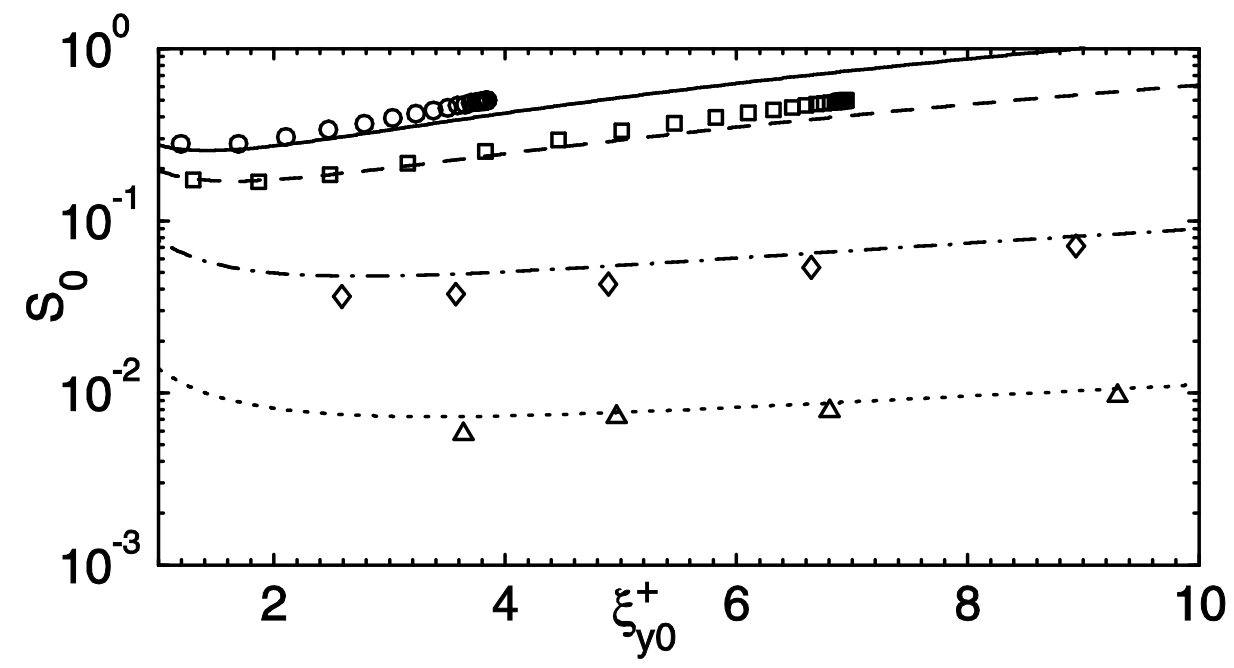

Figure 6. Fluid shear stress at the bed versus take-off velocity at large Stokes numbers. The symbols are the same as in Fig. 2. The lines are the analytical approximations to the fluid shear stress at the bed (Eq. 27) with $K=1.5$ and St $=1000$ for $\sigma=5$ (solid), $\sigma=10$ (dashed line), $\sigma=100$ (dot-dashed line) and $\sigma=1000$ (dotted line).

For the fluid shear stress at the bed to be positive, the trajectory height must be greater than $y_{0}$, so, from Eq. 16, $\xi_{y 0}^{+} \geq\left(2 y_{0}\right)^{1 / 2}$ . In fact, for continuing saltation to exist, the height of the periodic trajectory should be greater than one diameter; so, in dimensionless terms,

$$
\xi_{y 0}^{+} \geq \sqrt{2}^{\cdot}
$$

The minimum of the fluid shear stress in Fig. 6 corresponds to the smallest Shields parameter for which continuing saltation is possible over a rigid, bumpy bed. The difference between the Shields parameter and the fluid shear stress at the bed is, indeed, the particle shear stress, which must be positive. Using Eqs. 16, 18, 21 and 24 in Eq. 27, and requiring that the derivative with respect to the take-off velocity be zero, permits the determination of the take-off velocity at the minimum. Its approximate expression is

$$
\xi_{y 0}^{+c}=\max \left\{\exp \left[1+\frac{1}{2} \frac{(A+D) \ln \left(2 y_{0}\right)}{A+D+2 E}\right], \sqrt{2}\right\},
$$

where we have assumed that

$$
\ln \xi_{y 0, c}^{+} \simeq 1-1 / \xi_{y 0, c}^{+}
$$

. This value, used in Eq. 27, gives the critical Shields

parameter $S_{c}^{*}$ at which continuing saltation ceases:

$$
S_{c}^{*}=\frac{K \kappa^{2}}{\sigma\left[2 \ln \xi_{y 0}^{+c}-\ln \left(2 y_{0}\right)\right]^{2}}\left(\frac{A+D}{2} \xi_{y 0}^{+c}+E\right)^{2} .
$$

Equations 28 and 29 indicate that the product of this critical Shields number and the density ratio is only a function of $E$. We anticipate that Eqs. 28 and 29 are valid also for continuing saltation over erodible beds. There is only a small quantitative difference between the rigid and the erodible beds, due to the different values of the numerical coefficients $a, b, a_{y}$ and $b_{y}$ in the rebound relations, which determine the values of $A_{A}$ and ${ }_{D}$. Figure 7 shows the comparison between values of this product obtained numerically 
and analytically versus $E$. Also shown are the experimental values of the product for the initiation of particle motion over erodible beds obtained in the experiments of Meyer-Peter \& Müller (1948; $\sigma=2.5$ and St between 1000 and 32000), Creyssels et al. (2009; $\sigma=2500$ and St = 3000) and Burr et al. (2015; $\sigma$ between 80 and 200 and St between 4000 and 8500). Only data for hydrodynamically rough beds have been taken from the experiments. The predicted values of $S_{c}{ }^{*}$ for density ratios larger than 50 are close to the values of the minimum Shields number for particle motion, indicating that there is a transition from no motion to continuing saltation. In contrast, the aquatic data of Meyer-Peter \& Müller (1948), for which the Shields parameter is less than $S_{c}{ }^{*}$, indicate that there the motion is not characterized by continuing saltation. In other words, the mean motion of the fluid is not enough to sustain the particle transport. Indeed, in that case, turbulence bursts are responsible for the particle motion, which is characterized by intermittency (Ancey et al. 2008, Lajeunesse et al. 2010, Radice et al. 2009, Singh et al. 2009).

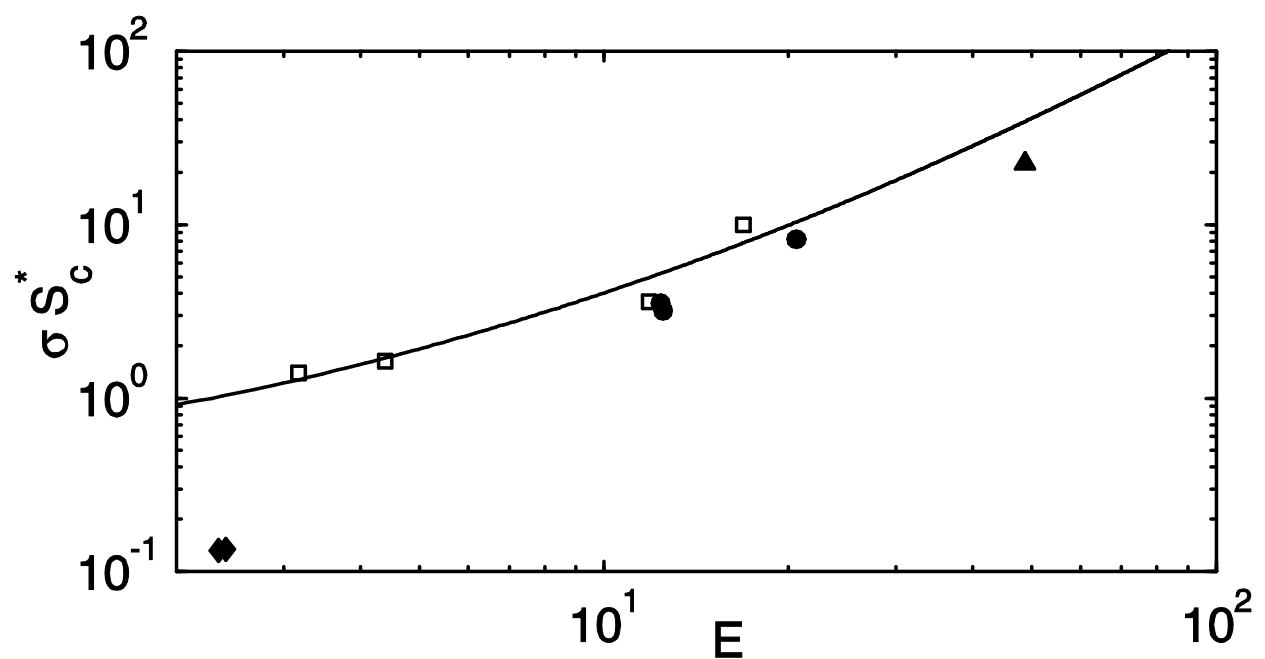

Figure 7. Product of the critical Shields parameter and the density ratio for continuing saltation versus the quantity $E$, as obtained from the analytical approximation Eq. 29 (line) and the numerical integrations for the periodic trajectories at large Stokes numbers (hollow squares). Also shown are the experimental values (solid symbols) of the product of the minimum Shields number and the density ratio for the initiation of steady particle motion obtained by Meyer-

Peter and Müller (1948, diamonds), Creyssels et al. (2009, triangles) and Burr et al. (2015, circles).

\subsection{Horizontal particle flux}

Given the Shields parameter $S^{*}$, the particle shear stress at the bed is

$$
s_{0}=S^{*}-K \frac{\kappa^{2}}{\sigma\left[\ln \left(H / y_{0}\right)\right]^{2}} \bar{U}^{2} .
$$

Unlike the fluid shear stress, the particle shear stress at the bed is influenced by the Shields parameter. Then, from Eqs. 9, 10 and 17,

$$
c_{0}^{+}=\frac{s_{0}}{\xi_{y 0}^{+}\left(\xi_{x 0}^{-}-\xi_{x 0}^{+}\right)} .
$$

Equations 30 and 31 relate the take-off velocity, the concentration, and the Shields parameter or, alternatively, the depth-averaged fluid velocity, the concentration, and the Shields parameter. The horizontal particle flux per unit width of the bed can then be calculated as

$$
Q=c_{0}^{+} L \xi_{y 0}^{+}
$$


Figures $8 \mathrm{a}$ and $8 \mathrm{~b}$ show the comparison between the horizontal particle flux evaluated from the full numerical integration of the equations for the periodic trajectories at large Stokes numbers and that obtained from the approximate analytical approach for two extreme values of the density ratio at different Shields parameters, with the flux linearly increasing with the Shields parameter.

(a)

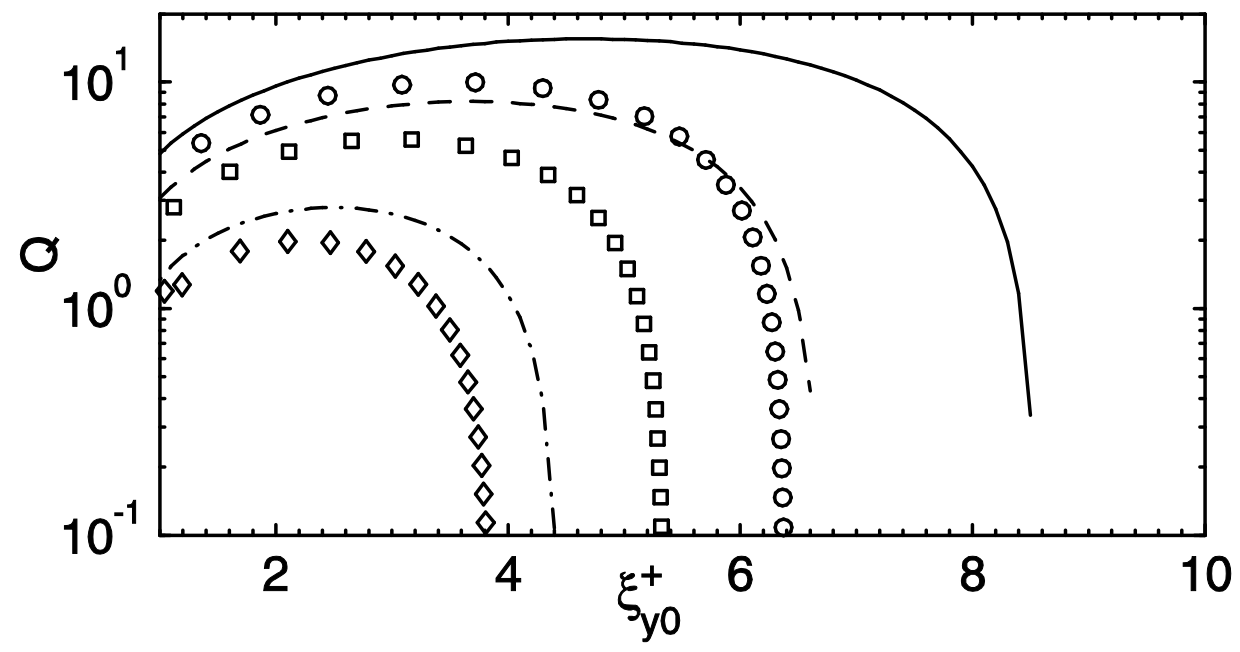

(b)

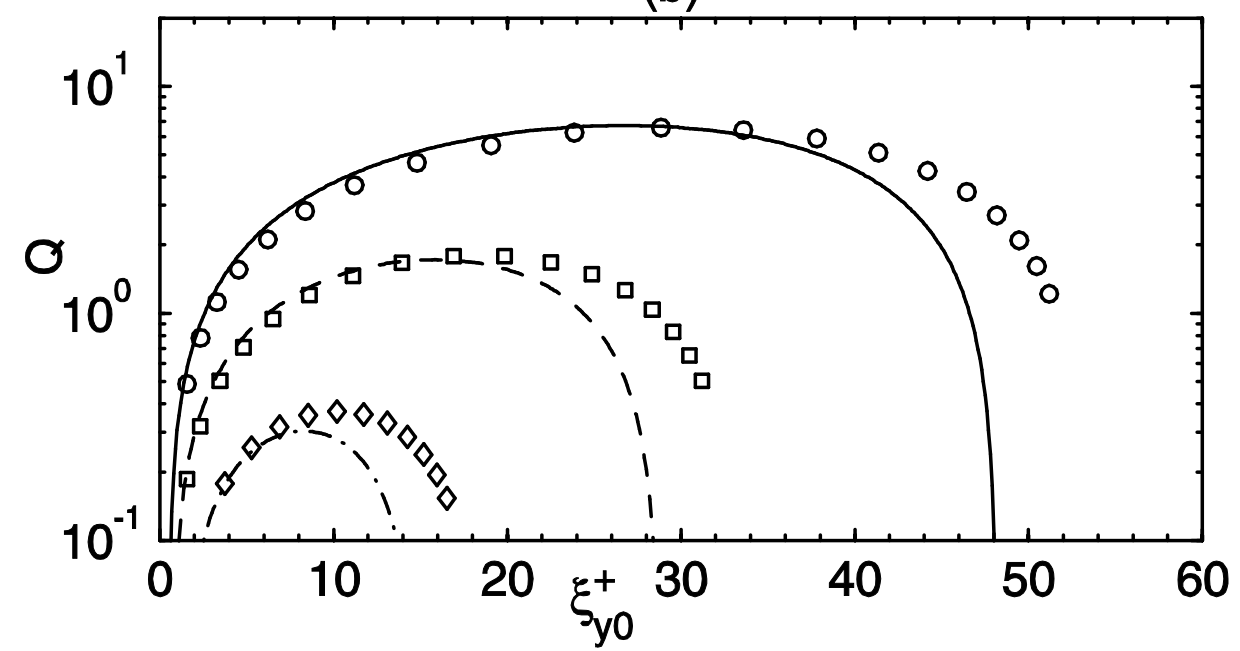

Figure 8. Horizontal particle flux versus take-off velocity for periodic saltation over rigid beds when St = 1000 and: (a) $\sigma=5$ and $S^{*}=0.3$ (triangles and dotted line), 0.5 (diamonds and dot-dashed line), 0.8 (squares and dashed line), 1.1 (circles and solid line); and (b) $\sigma=1000$ and $S^{*}=0.02$ (diamonds and dot-dashed line), 0.04 (squares and dashed line) and 0.08 (circles and solid line). The symbols are the numerical solutions for the periodic trajectories and the lines are the analytical approximation to the particle flux (Eq. 32).

As already noticed in the experiments (Ho et al. 2011) and in the analysis of Jenkins \& Valance (2014) on Aeolian transport over rigid beds, there is a maximum horizontal particle flux $Q_{\max }$ that the fluid can sustain at a given Shields parameter: the maximum transport capacity of the flow. Our analysis suggests that this observation holds irrespective of the density ratio. We can determine the value of the take-off velocity, ${ }_{\hat{\xi}_{y 0}^{+}}$, that corresponds to $Q_{\max }$ by requiring the derivative of Eq. 32 with respect to $\xi_{y 0}^{+}$to be zero,

using Eqs. 16, 18, 21, 24, 26, 30 and 31. This results in the condition 


$$
\begin{aligned}
\frac{\sigma S^{*}}{K \kappa^{2}}= & {\left[\left(\frac{A+D}{2} \hat{\xi}_{y 0}^{+}+E\right)(A+D) \hat{\xi}_{y 0}^{+}+\left(\frac{A+D}{2} \hat{\xi}_{y 0}^{+}+E\right)^{2}\right]\left[\ln \left(\frac{\hat{\xi}_{y 0}^{+2}}{2 y_{0}}\right)\right]^{-2} } \\
& -4\left(\frac{A+D}{2} \hat{\xi}_{y 0}^{+}+E\right)^{2}\left[\ln \left(\frac{\hat{\xi}_{y 0}^{+2}}{2 y_{0}}\right)\right]^{-3}
\end{aligned}
$$

With this and Eqs. 24, 26, 30, 31 and 32, the maximum transport capacity $Q_{\max }$ can then be calculated as

$$
\sigma Q_{\max }=\frac{A+D}{A-D}\left[\sigma S^{*}-K \frac{\kappa^{2}}{\left[2 \ln \hat{\xi}_{y 0}^{+}-\ln \left(2 y_{0}\right)\right]^{2}}\left(\frac{A+D}{2} \hat{\xi}_{y 0}^{+}+E\right)^{2}\right] \hat{\xi}_{y 0}^{+}
$$

Equation 34 provides a relation between $Q_{\max }, S^{*}$, and $\sigma$, once $\hat{\xi}_{y 0}^{+}$is determined as a function of $S^{*}$ from Eq. 33. With $\hat{\xi}_{y 0}^{+}$, all the quantities that characterize the periodic trajectory at the maximum transport capacity can easily be obtained from the equations introduced in this section. 


\section{Approximate analytical solution: periodic saltation over an erodible bed}

In continuing, steady saltation over erodible beds, there is, in principle, an unlimited supply of material that can be incorporated in the flow. The horizontal particle flux may be calculated for all impact velocities. If the flux corresponding to half the critical impact velocity resides to the right of the maximum, an increment of impact velocity to its left results in an increase in flux, and erosion from the bed. With continued migration of the impact velocity to the left, the increase in horizontal particle flux and erosion continues until the maximum horizontal flux is achieved. The horizontal particle flux $Q$ is always equal to $Q_{\max }$ of Eq. 34, with $\hat{\xi}_{y 0}^{+}$calculated from Eq. 33, when it is less than $\xi_{c} /(2 B)$, and equal to $\xi_{c} /(2 B)$ otherwise. We refer to

these cases as unlimited and splash-limited saltation, respectively. The condition $\hat{\xi}_{y 0}^{+}=\xi_{c} /(2 B)$

is the

constraint assumed by Jenkins \& Valance (2014) in their analysis of Aeolian transport over erodible beds. Aeolian transport over an erodible bed is splash limited, while aquatic transport is not.

The boundary between unlimited and splash-limited saltation is, from Eq. 33 with $\hat{\xi}_{y 0}^{+}=\xi_{c} /(2 B)^{\prime}$

at the Shields parameter

$$
\begin{aligned}
\frac{\sigma S_{s}^{*}}{K \kappa^{2}}= & {\left[\left(\frac{A+D}{2} \frac{\xi_{c}}{2 B}+E\right)(A+D) \frac{\xi_{c}}{2 B}+\left(\frac{A+D}{2} \frac{\xi_{c}}{2 B}+E\right)^{2}\right]\left[\ln \left(\frac{\xi_{c}{ }^{2}}{8 B^{2} y_{0}}\right)\right]^{-2} } \\
& -4\left(\frac{A+D}{2} \frac{\xi_{c}}{2 B}+E\right)^{2}\left[\ln \left(\frac{\xi_{c}^{2}}{8 B^{2} y_{0}}\right)\right]^{-3} .
\end{aligned}
$$

Unlimited saltation takes place for Shields parameters in the range from $S_{c}^{*}{ }_{S_{s}^{*}}^{\text {; splash-limited }}$ saltation occurs for Shields parameters larger than $S_{s}^{*}$ As demonstrated in Jenkins \& Valance (2014), the two regimes are characterized by different scaling of the various quantities with the Shields parameter. In particular, the horizontal particle flux scales with the $3 / 2$ power of the difference between the Shields parameter and $S_{c}^{*}$ in unlimited saltation (it is the scaling of the maximum transport capacity over rigid beds); while it scales linearly with the Shields parameter in the splash-limited case. Figure 9 shows the comparison between the analytical horizontal particle flux as a function of the Shields parameter for periodic saltation over erodible beds and the results of the numerical solutions for the periodic trajectories for large Stokes numbers. To determine the latter, we use the same criterion of the analytical solution: the particle flux at a given Shields parameter is the maximum in the curves of Fig. 8 - calculated through interpolation - unless the corresponding impact velocity exceeds ; in that case, we take the horizontal $\xi_{c} / 2$

particle flux to be the value to the left of the maximum at which the impact velocity is exactly $\xi_{c} / 2$ 
Also shown in Fig. 9 are the experimental values obtained by Creyssels et al. (2009) and Ho et al. (2011) for sand in air. The analytical solution for $\sigma=2500$ and $\mathrm{St}=3000$, as in the experiments, is indistinguishable from that for $\sigma=1000$ and $\mathrm{St}=1000$. As in the numerical simulations of Durán et al. (2012), the horizontal particle flux scales linearly with the Shields parameter in the Aeolian case $(\sigma=1000)$ - splash-limited saltation - and is roughly proportional to the Shields parameter to the power of $3 / 2$ in the nearly aquatic case $(\sigma=5)$ - unlimited saltation. Intermediate values of the density ratio $(\sigma=100)$ are characterized by a mixed behaviour: the flux follows the unlimited saltation scaling close to the threshold, and the splash-limited saltation scaling for larger values of the Shields parameter. Our interpretation for the linear relationship between the horizontal particle flux and the Shields parameter in Aeolian saltation over erodible beds is based on the same argument as Durán et al. (2012): the splash process is the key mechanism that controls the saltation. The only difference is that we take the particle horizontal velocity proportional to the critical impact velocity, whereas Durán et al. suggest that it scales with the fluid horizontal velocity at the transport threshold.

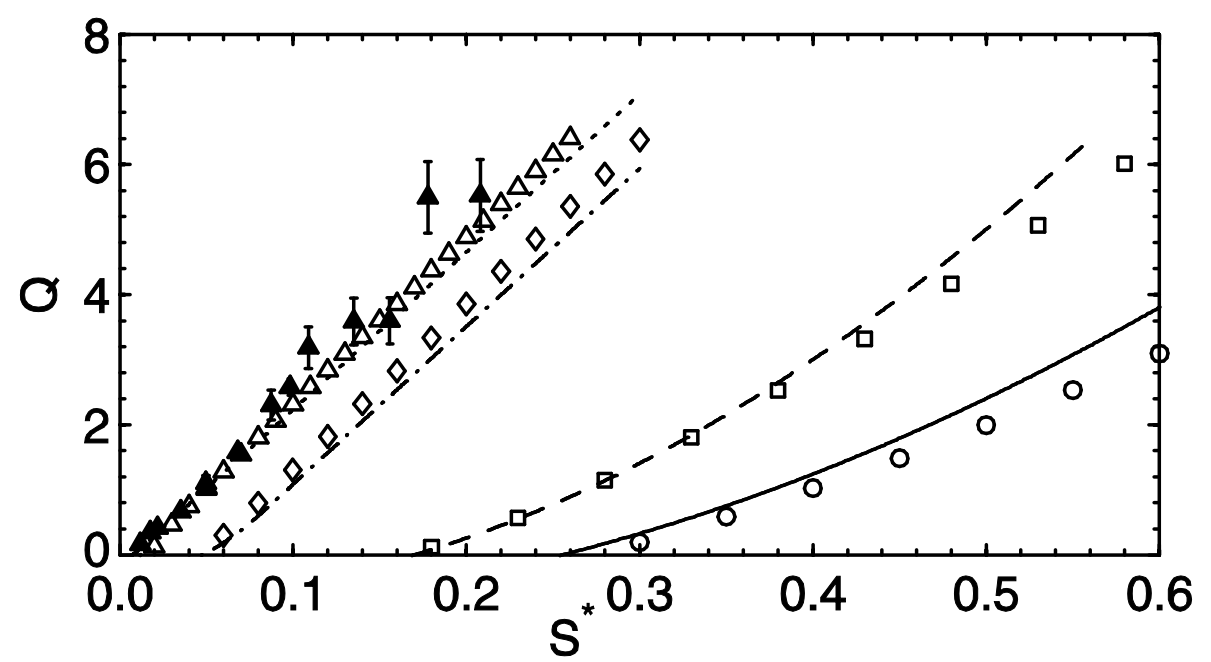

Figure 9. Horizontal particle flux versus Shields parameter for saltation over erodible beds for St $=1000$, when $\sigma=5$ (circles and solid line), $\sigma=10$ (squares and dashed line), $\sigma=100$ (diamonds and dot-dashed line) and $\sigma=1000$ (triangles and dotted line). The hollow symbols are the numerical solutions for the periodic trajectories and the lines are the analytical approximation to the particle flux (Eq. 34). Also shown are the experimental results of Creyssels et al. (2009) and Ho et al. (2011) (solid triangles) with the relative error bars.

Saltation alone takes place when inter-particle collisions above the bed are not likely. This is no longer true when twice the height of the trajectory $H$ is greater than the mean free path of kinetic theory, $\sqrt{2} / 12 c_{0}$

(Chapman \& Cowling 1970, Pasini \& Jenkins 2005), where The height of the trajectory and the $c_{0}=c_{0}^{+}+c_{0}^{-}$

concentration at the bed can be calculated from the relations derived in the previous section, with the takeoff velocity equal to the minimum between ${ }_{\xi_{y 0}^{+}}$, calculated from Eq. 33 and $\xi_{c} /(2 B)$. This provides an upper limit for the existence of a pure saltation regime in terms of the Shields parameter as a function of the density ratio at a given Stokes number. Above this limit, collisional suspension begins to take place (Berzi 2013, Jenkins \& Hanes 1998, Pasini \& Jenkins 2005).

Figure 10 shows a regime map in terms of Shields parameter versus density ratio for a Stokes number of 1000. At large density ratios (Aeolian transport), there is a rather abrupt transition from no motion to continuing saltation, and the latter is essentially limited by the splash. 
Also the range of Shields parameter in which continuing saltation exists is significant. This confirms that saltation is the main mode of transport for sand in air. On the other hand, at small density ratios (aquatic transport), the intermittent motion is dominant up to large Shields parameters, at which there is a rather abrupt transition to collisional suspension. In this case, continuing saltation, not limited by the splash, is only marginally relevant. Intermediate values of the density ratio, typical of many extra-terrestrial environments, are more complicated: intermittent motion, unlimited and splash-limited saltation and collisional suspension seem to play equally significant roles in the transport of sediment.

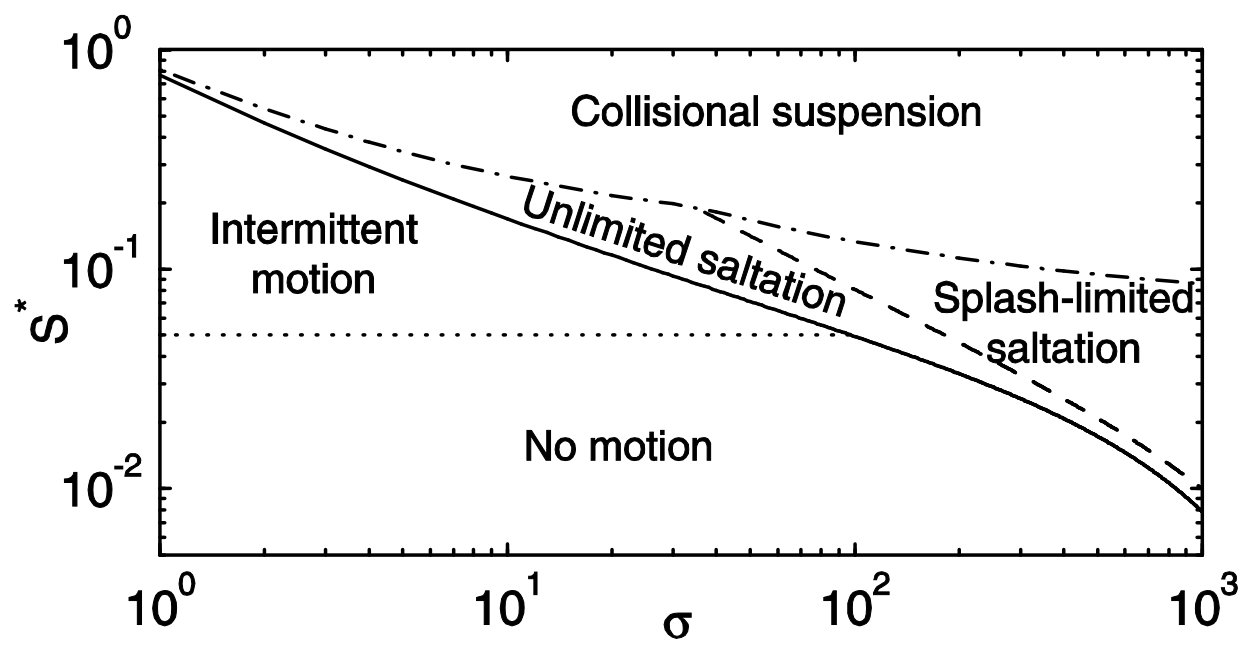

Figure 10. Regime map for St $=1000$. The boundary between the regimes of intermittent motion and no motion is qualitatively determined (Durán et al. 2012).

\section{Conclusion}

We have presented an approximate analytical analysis of particle periodic motion over hydrodynamically rough beds. The analysis was based on the calculation of approximate solutions for average, periodic trajectories of particles that are accelerated by the turbulent shearing of a fluid, between collisions with the bed. We have focused on the case in which the mean fluid motion is strong enough to sustain the saltation of the particles, as continuing rather than intermittent, as often seen in weak bed-load transport of particles in water.

From these solutions, we have determined the relations between the horizontal particle flux, the strength of the shearing flow and the particle take-off velocity over a range of the grain-to-fluid mass density ratios that vary between those for sand in air and sand in water, in saltation over rigid, bumpy and erodible beds. We have focused on large values of the Stokes number, where collisions with the bed are not influenced by the fluid. We have also shown how to extend the analysis to smaller values of the Stokes number.

For saltation over rigid, bumpy beds, we have predicted that there is range of particle flux that the fluid can sustain at a given Shields parameter, irrespective of the density ratio and the Stokes number. That range presents a maximum, which corresponds to the maximum transport capacity of the flow, before particles begin to be deposited and an erodible bed develops. To our knowledge, these findings have been previously demonstrated in the case of Aeolian transport only. We have also found that the particle trajectory and the fluid shear stress at the bed are essentially independent of the Shields parameter, unlike the particle shear stress and concentration.

For saltation over erodible beds, there is only one horizontal particle flux associated with a given Shields parameter. The analytical solution indicates that this flux scales linearly with the Shields parameter in Aeolian transport, while it is roughly proportional to the Shields number to the power of $3 / 2$ near aquatic transport. These predictions are in agreement with the scaling laws drawn from sand transport experiments in air and water. 
We have also highlighted that saltation regimes in air and water are different in nature: Aeolian saltation is limited by the splash, while the aquatic saltation is not. In the latter case, the impact velocity of the saltating particles is actually too weak to trigger the splash and, as a consequence, the particle flux is limited by the maximum transport capacity of the system. Interestingly, for intermediate values of the density ratio (typically between 30 and a few hundred), we have found a crossover regime: the particle flux is proportional to $S^{* 3 / 2}$ close to the threshold and linear in $S^{*}$ at larger values of the Shields parameter. In other words, for these intermediate values of $\sigma$, we observe a transition between an unlimited saltation regime at small Shields parameters to a splash-limited saltation regime at larger Shields parameters. This crossover regime is expected to be relevant for sediment transport in extra-terrestrial atmospheres such as on Venus and Titan, where the density ratios are about 40 and 200, respectively (Burr et al. 2015, Iversen \& Greeley 1987, Iversen \& White 1982).

We have determined the domain of existence of the continuing saltation regime in terms of the Shields parameter as a function of the density ratio at a given Stokes number. The lower limit (i.e., the critical Shields parameter below which continuing saltation ceases) is found to decrease with the density ratio. In Aeolian transport, the critical Shields number provides a good estimate of the threshold for incipient transport; while, in aquatic transport, it overestimates the transport threshold by a large amount. We have explained this discrepancy by noting that in the aquatic case, intermittent turbulence bursts play an important role, and can transport particles even when the mean motion is not strong enough to sustain continuing saltation. We have also provided an upper limit for the saltation regime, above which collisional suspension takes place.

In summary, we have shown that the description of particle transport in terms of periodic saltation is relevant for a wide range of physical systems. In addition, the simplicity of this description permits the derivation of approximate analytical expressions for key features of the sediment transport, including the height and length of the particle trajectories, the fluid shear stress, and the horizontal particle flux. This study could be continued in different directions: it would be interesting to look at the case of hydrodynamically smooth beds, and further work should be done to describe the motion in the intermittent regime.

This research was supported in part by the National Science Foundation under grant no. NSF PHY11-25915 to the Kavli Institute of Theoretical Physics. 
Appendix A. Stokes dependency in the rebound relations and non-linear drag in the horizontal momentum balance

For arbitrary values of the Stokes number, when the vertical drag is neglected, Eqs. 15 and 17 give

$$
e_{y}=\frac{\mathrm{St} \xi_{y 0}^{+}+62}{\mathrm{St} \xi_{y 0}^{+}-62} .
$$

When $\xi_{y 0}^{+}>1240 / \mathrm{St}$

does not substantially affect the collisions with the bed. Equation A1 shows that

$$
\xi_{y 0}^{+} \geq \frac{62}{\mathrm{St}}
$$

Then, the angle of impact is

$$
\sin \theta=\frac{a_{y}}{e_{y}+b_{y}} .
$$

The horizontal particle velocity at the end of the trajectory is, upon taking

$$
\sin \theta \approx \tan \theta^{\prime}
$$

$$
\xi_{x 0}^{-}=\xi_{y 0}^{+} \frac{e_{y}+b_{y}}{a_{y}}
$$

In this event, the impact velocity is

$$
\xi_{0}^{-}=\xi_{y 0}^{+}\left[\left(\frac{e_{y}+b_{y}}{a_{y}}\right)^{2}+1\right]^{1 / 2}
$$

From Eq. 14, we calculate the absolute value of the velocity after the rebound,

$$
\xi_{0}^{+}=\left(a-b \frac{a_{y}}{e_{y}+b_{y}}\right) \xi_{0}^{-}-\frac{62}{\mathrm{St}}\left(1+a-b \frac{a_{y}}{e_{y}+b_{y}}\right) .
$$

Given that $e$ is now known, the angle of impact has been determined; hence, the horizontal velocity after the rebound is 


$$
\xi_{x 0}^{+}=\left[\left(\xi_{0}^{+}\right)^{2}-\left(\xi_{y 0}^{+}\right)^{2}\right]^{1 / 2}
$$

Appendix B. Relation between the fluid shear stress at the bed and the depth-averaged fluid horizontal velocity

If we take the horizontal fluid velocity to obey the logarithmic law,

$$
\frac{U}{u^{*}}=\frac{1}{\kappa} \ln \left(\frac{y}{y_{0}}\right)
$$

with
\[ u^{*}=\left(\sigma S_{0}\right)^{1 / 2} \text {, the shear velocity. Integrating, } \]

$$
\frac{\bar{U}}{u^{*}} H \simeq \int_{y_{0}}^{H} \frac{U}{u^{*}} d y=\int_{y_{0}}^{H} \frac{1}{\kappa} \ln \left(\frac{y}{y_{0}}\right) d y=\frac{y_{0}}{\kappa}\left[\frac{H}{y_{0}} \ln \left(\frac{H}{y_{0}}\right)-\frac{H}{y_{0}}+1\right] \simeq \frac{H}{\kappa} \ln \left(\frac{H}{y_{0}}\right)
$$

Finally,

$$
S_{0} \simeq \frac{\kappa^{2}}{\sigma}\left[\ln \left(\frac{H}{y_{0}}\right)\right]^{-2} \bar{U}^{2}
$$




\section{REFERENCES}

Abbot JE, Francis JRD. 1977. Saltation and Suspension Trajectories of Solid Grains in a Water Stream. Phil. Trans. R. Soc. A 284:225-254

Andreotti B. 2004. A two-species model of aeolian sand transport. J. Fluid Mech. 510:47-70

Ancey C, Bigillon F, Frey P, Lanier J, Ducret R. 2002. Saltating motion of a bead in a rapid water stream. Phys. Rev. E. 66(3):036306

Ancey C, Davison a. C, Böhm T, Jodeau M, Frey P. 2008. Entrainment and motion of coarse particles in a shallow water stream down a steep slope. J. Fluid Mech. 595:83-114

Anderson RS, Haff PK. 1988. Simulation of Eolian Saltation. Science 241:820-823

Bagnold RA. 1941. The Physics of Blown Sand and Desert Dunes. New York: Methuen.

Bagnold RA. 1966. An approach to the sediment transport problem from general physics. US Geological Survey Professional Paper 422-I:37.

Beladjine D, Ammi M, Oger L, Valance A. 2007. Collision process between an incident bead and a threedimensional granular packing. Phys. Rev. E. 75(6):061305

Berzi D. 2013. Transport Formula for Collisional Sheet Flows with Turbulent Suspension. J. Hydraul. Eng. ASCE. 139(4):359-63

Berzi D, Fraccarollo L. 2013. Inclined, collisional sediment transport. Phys. Fluids. 25(10):106601

Burr DM, Bridges NT, Marshall JR, Smith JK, White BR, Emery JP. 2015. Higher-than-predicted saltation threshold wind speeds on Titan. Nature. 517:60-63

Chapman S, Cowling TG. 1970. The Mathematical Theory of Non-Uniform Gases. Cambriddge University Press.

Crassous J, Beladjine D, Valance A. 2007. Impact of a Projectile on a Granular Medium Described by a Collision Model. Phys. Rev. Lett. 99:248001

Creyssels M, Dupont P, El Moctar AO, Valance A, Cantat I, et al. 2009. Saltating particles in a turbulent boundary layer: experiment and theory. J. Fluid Mech. 625:47

Drake TG, Shreve RL, Dietrich WE, Whiting PJ, Leopold LB. 1988. Bedload transport of fine gravel observed by motion-picture photography. J. Fluid Mech. 192(-1):193

Drew DA. 1975. Turbulent sediment transport over a flat bottom using momentum balance. J. Appl. Mech. 42:38-44.

Durán O, Claudin P, Andreotti B. 2011. On aeolian transport: Grain-scale interactions, dynamical mechanisms and scaling laws. Aeolian Res. 3:243-270

Durán O, Andreotti B, Claudin P. 2012. Numerical simulation of turbulent sediment transport, from bed load to saltation. Phys. Fluids. 24:103306

Fernandez Luque R, van Beek R. 1976. Erosion and transportof bed-load sediment. J. Hydr. Res. 14(2):127144 
Foucaut J-M, Stanislas M. 1997. Experimental study of saltating particle trajectories. Exp. Fluids 22(4):321326

Gersten K, Schlichting H. 2000. Boundary-Layer Theory. Berlin: Springer

Greeley R, Iversen J, Leach R, Marshall J, Williams S, White B. 1984. Windblown sand on Venus - Preliminary results of laboratory simulations. Icarus. 57:112-24

Ho TD, Dupont P, Ould El Moctar A, Valance A. 2012. Particle velocity distribution in saltation transport. Phys. Rev. E. 85(5):052301

Ho TD, Valance A, Dupont P, Ould El Moctar A. 2011. Scaling laws in aeolian sand transport. Phys. Rev. Lett. 106:4-7

Ho TD, Valance A, Dupont P, Ould El Moctar A. 2014. Aeolian sand transport: Length and height distributions of saltation trajectories. Aeolian Res.. 12:65-74

Hsu T-J, Jenkins JT, Liu PL-F. 2003. On two-phase sediment transport: Dilute flow. J. Geophys. Res. 108(C3):3057

Iversen JD, Greeley R. 1987. Aeolian saltation threshold: the effect of density ratio. Sedimentology 34(4):699-706

Iversen JD, Rasmussen KR. 1999. The effect of wind speed and bed slope on sand transport. Sediment. 46:723-731

Iversen JD, White BR. 1982. Saltation threshold on Earth, Mars and Venus. Sediment. 29(1):111-19

Jenkins JT, Cantat I, Valance A. 2010. Continuum model for steady, fully developed saltation above a horizontal particle bed. Phys. Rev. E 82:020301

Jenkins J, Hanes D. 1998. Collisional sheet flows of sediment driven by a turbulent fluid. J. Fluid Mech. 370:29-52

Jenkins JT, Valance A. 2014. Periodic trajectories in aeolian sand transport. Phys. Fluids 26(7):073301

Kok JF, Renno NO. 2009. A comprehensive numerical model of steady state saltation (COMSALT). J. Geophis. Res. 114:D17204

Kok JF, Parteli EJR, Michaels TI, Karam DB. 2012. The physics of wind-blown sand and dust. Rep. Progr. Phys. 75(10):106901

Lajeunesse E, Malverti L, Charru F. 2010. Bed load transport in turbulent flow at the grain scale: Experiments and modeling. J. Geophys. Res. 115:F04001

McTigue DF. 1981. Mixture theory for suspended sediment transport. J. Hydr. Div. ASCE 107:659-673.

Meyer-Peter E., Müller R. 1948. Formulas for Bed-Load Transport. Proc. Second Congress IAHR, Stockolm, Sweden.

Nalpanis P, Hunt JCR, Barrett CF. 1993. Saltating particles over flat beds. J. Fluid Mech. 251:661-685

Nelson JM, Shreve RL, McLean SR, Drake TG. 1995. Role of near-bed turbulencein bed load transport and bed form mechanics. Water Resour. Res. 31(8):2071-86

Niño Y, García M. 1998. Experiments on saltation of sand in water. J. Hydraul. Eng. ASCE 124(10):1014-25 
Oger L, Ammi M, Valance a, Beladjine D. 2005. Discrete Element Method studies of the collision of one rapid sphere on 2D and 3D packings. Eur. Phys. J. E 17(4):467-76

Owen, PR. 1964. Saltation of uniform grains in air. J. Fluid Mech. 20:225-242

Pasini JM, Jenkins JT. 2005. Aeolian transport with collisional suspension. Philos. Trans. A. Math. Phys. Eng. Sci. 363(1832):1625-1646

Radice A, Ballio F, Nikora V. 2009. On statistical properties of bed load sediment concentration. Water Resour. Res. 45(6):1-8

Sauermann G, Kroy K, Herrmann HJ. 2001. A Continuum Saltation Model for Sand Dunes. Phys. Rev. E 64:031305

Singh A, Fienberg K, Jerolmack DJ, Marr J, Foufoula-Georgiou E. 2009. Experimental evidence for statistical scaling and intermittency in sediment transport rates. J. Geophys. Res. 114(1):1-16

Ungar JE, Haff PK. 1987. Steady state saltation in air. Sediment. 34(2):289-299

Valance A, Rasmussen KR, El Moctar AO, Dupont P. 2015. The physics of Aeolian sand transport. Compt. Rend. Phys. 16(1):105-117

van Rijn LC. 1984. Sediment transport, part I: bed load transport. J. Hydraul. Eng. ASCE 110(10):1431-56

White BR. 1979. Soil transport by winds on Mars. J. Geophys. Res. 84(B9):4643-51

Yang F-L, Hunt ML. 2006. Dynamics of particle-particle collisions in a viscous liquid. Phys. Fluids.

18(12):121506 